\title{
Tecnologia Educativa para capacitação de familiares cuidadores de adultos mais velhos dependentes
}

\section{Educational Technology in training family caregivers of older people}

\author{
Luiziany Pontes Rios Osterne (1) \\ Zélia Maria de Sousa Araújo Santos (2) \\ Maria Regina Teixeira Ferreira Capelo (3) \\ July Grassiely de Oliveira Branco (4) \\ Emanuelly Pontes Rios Osterne (5) \\ Manoel Pereira de Sousa Filho (6)

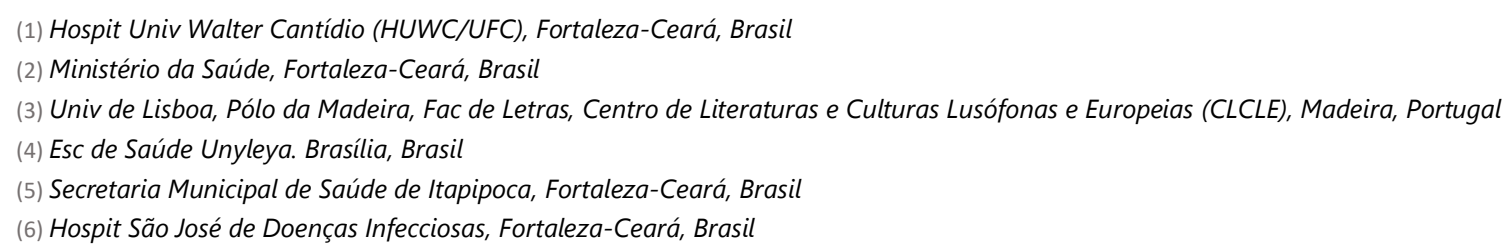

Recebido: 18/03/2021; Revisto: 17/05/2021; Aceite: 28/05/2021.

\begin{abstract}
Resumo
Contexto e Objetivo: Cuidar de adultos mais velhos dependentes no domicílio é uma realidade cada vez mais frequente na atual sociedade envelhecida, advindo daí a necessidade de dotar os cuidadores com práticas e saberes que, tradicionalmente, não estavam nos domínios das famílias. Esta investigação tem como objetivo analisar a eficácia de uma Tecnologia Educativa na capacitação de familiares cuidadores de adultos mais velhos dependentes em ambiente domiciliar. Métodos: Participaram 12 familiares cuidadores que acompanharam adultos mais velhos internados numa instituição hospitalar de referência em traumas, situada em Fortaleza-Ceará-Brasil, um mês após o retorno ao domicílio. Tratou-se de um estudo qualitativo assente na investigação-ação mediante construção, aplicação e avaliação de uma tecnologia educativa (TE). A TE construída para o efeito, teve por base a Teoria das Necessidades Humanas Básicas de Maslow e os pressupostos da educação para a saúde. Resultados: Oito familiares cuidadores relataram a experiência prévia no cuidado aos adultos mais velhos no domicílio e quatro revelaram um conhecimento superficial sobre o cuidar. A aquisição de conhecimento mediado pela aplicação da Tecnologia Educativa conduziu à ocorrência de mudanças no atendimento às necessidades humanas básicas de adultos mais velhos no cuidado domiciliar, designadamente, compreensão do processo de envelhecimento, adaptação do ambiente físico, adequação da alimentação, tolerância, resgate da autonomia e envolvimento da família. Conclusões: A aplicação da TE teve impacto na práxis do familiar cuidador, materializado no suprimento das necessidades básicas humanas da pessoa idosa cuidada. Esta ferramenta, desponta como complemento útil para o reforço das competências do cuidador informal do adulto mais velho dependente.
\end{abstract}

Palavras-Chave: Cuidador informal; Saúde do adulto mais velho; Tecnologia Educativa; Educação para a Saúde.

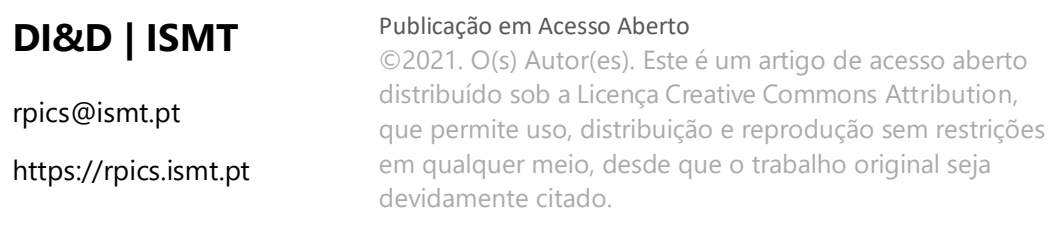

Maria Regina Teixeira Ferreira Capelo

Campus Universitário da Penteada, Piso 1, Gabinete 1001, 9000-208, Funchal, Madeira, Portugal

E-mail: m.regina.capelo@gmail.com 


\begin{abstract}
Background and Aim: Caring for dependent older adults at home is an increasingly frequent reality in today's ageing society, resulting in the need to provide careers with the skills and knowledge which were not traditionally part of the family realm. This research aims to analyze the effectiveness of an Educational Technology in the training process of careers of dependent older adults at home. Method: Participants were 12 family caregivers who accompanied older adults admitted to a reference hospital in trauma, located in Fortaleza-Ceará-Brazil, a month after returning home. Eight family caregivers reported previous experience caring for older adults at home, and four revealed to have a superficial knowledge of the matter. This was a qualitative study based on action research through the creation, application and assessment of an educational program. The Educational Technology built for this purpose was based on the Theory of Basic Human Needs and the assumptions of health education. Results: Knowledge acquisition mediated by the application of Educational Technology led to changes in providing basic human needs of older adults' in-home care, namely the understanding of the ageing process, adaptation of the physical environment, nutritional adequacy, tolerance, autonomy and family involvement. Conclusions: The use of Educational Technology had an impact on the praxis of the family caregiver through the supply of the basic human needs of the older person being cared for. This tool emerges as a useful complement to strengthen the abilities of the informal caregiver of the dependent older adult.
\end{abstract}

Keywords: Informal caregiver; Health of the older adult; Educational Technology; Health Education.

\title{
Introdução
}

À medida que a população envelhece, um número crescente de pessoas idosas dependentes aumenta em todo o mundo (World Health Organization [WHO], 2015). Conceitualmente, o envelhecimento é definido como um processo complexo, progressivo e irreversível, associado ao acúmulo de uma variedade de danos moleculares e celulares, que ocorre ao longo do ciclo vital, relacionado com o aparecimento de doenças crónicas que podem incapacitar e conduzir o indivíduo à dependência funcional ou até à morte (Galado, 2018; Santos et al., 2017; WHO, 2015). Portanto, o envelhecimento está relacionado com o declínio geral das capacidades físicas e mentais em que o indivíduo se pode apoiar, isto é, das suas capacidades intrínsecas (WHO, 2015). No entanto, o surgimento de patologias não é linear, pois o fenômeno decorre de forma individual na relação que o indivíduo estabelece com o contexto envolvente (Organização Pan-Americana da Saúde/Organização Mundial de Saúde, 2018; WHO, 2015). Em contrapartida, numa perspetiva mais positiva, o envelhecimento é encarado como sabedoria, razão e serenidade (Fechine \& Trompieri, 2012).

A literatura sublinha a existência de um número crescente de idosos com dependência funcional para realizar a higiene pessoal, as tarefas domésticas e até a mobilidade (Cruz et al., 2010; Galado, 2018; Landeiro et al., 2016; Pereira \& Duque, 2017). A situação ideal consiste na manutenção da autonomia e da independência pelo maior tempo possível porque, o idoso, quanto mais independente funcional estiver, menos cuidados requer (Pereira et al., 2014). Na atualidade muitos adultos mais velhos dependem de cuidadores, de quem o auxilie nas atividades quotidianas para as quais não dispõe de autonomia. Essas atividades incluem higiene corporal, locomoção, medidas de conforto, alimentação, elo entre o idoso, a família e a equipa de saúde, entre outras atividades inerentes ao ato de cuidar (Ministério da Saúde, 2008). Essas necessidades da pessoa idosa devem ser respeitadas e entendidas como prioritárias; isto é, o cuidador participa para supri-las, já que o idoso em situação de dependência parcial ou total não consegue promover efetivamente o autocuidado, encontrandose impossibilitado de satisfazer as suas necessidades básicas e de realizar as tarefas mais básicas de sobrevivência. Neste âmbito, o modelo teórico das Necessidades Humanas Básicas fundamentado na Hierarquia das Necessidades Humanas (Horta, 1979), usado amplamente na Enfermagem, e na Teoria da Motivação Humana, evidencia as necessidades de nível psicobiológico, psicossocial e psicoespiritual (Moraes 
et al., 2016). Essas necessidades são fundamentais para a manutenção do equilíbrio e devem ser atendidas e trabalhadas constantemente para que o indivíduo contribua para a manutenção de um estado de vida saudável. Baseada nessas demandas individuais, expressas nas teorias supramencionadas, cada pessoa idosa necessita de atenção específica para a manutenção da vida. Quando o adulto mais velho, dependente, não consegue realizar as ações básicas do dia a dia, são familiares, amigos, vizinhos ou outras pessoas não renumeradas pelos cuidados que prestam que assumem o papel de cuidador informal (Alves, 2018). As condições em que vive este grupo indica precariedade da função de cuidar, marcada por desigualdades, sobrecargas, adoecimentos e múltiplos problemas (Ceccon et al., 2021). Neste contexto, o despreparo para o cuidado, as dificuldades financeiras, o cerceamento da liberdade e os problemas de saúde física e mental são especificidades presentes na vida do cuidador de adultos mais velhos dependentes (Sousa et al., 2021). Por isso, torna-se entendível a resistência de alguns cuidadores em aceitarem a alta hospitalar dos familiares adultos mais velhos dependentes de cuidado, provavelmente por défice ou ausência de orientações para alta, bem como por carência de qualificação ou competência que Ihes permita atuar de forma eficiente, segura e individualizada perante a responsabilidade emergente, díspar das experiências proporcionadas por hospitais australianos através de programas que envolvem a capacitação de cuidadores informais para prestarem cuidados a adultos mais velhos após alta hospitalar (Slatyer et al., 2019). Assim sendo, a aplicação de uma Tecnologia Educativa dinamizadora do cuidado com idosos, baseada nos pressupostos da educação em saúde, poderá contribuir para minimizar o défice de conhecimento existente entre os cuidadores, considerando os benefícios do ganho da aquisição de conhecimento e o consequente impacto na prática de cuidar (Cardoso et al., 2018).

Uma das possibilidades para enfrentar o desafio de cuidar da pessoa idosa dependente em contexto comunitário é criar ações de educação para a saúde, através de tecnologia educativa focada no cuidado domiciliar, assente no desenvolvimento de ferramentas valorizadoras da escuta ativa e qualificada, na comunicação e na participação do indivíduo, protagonistas do cuidado, como agente transformador da realidade (Melo, 2016). A tecnologia educativa deve possibilitar a reflexão e a crítica para a construção do próprio conhecimento, apresentando-se como uma ferramenta útil no processo de aprendizagem e de adoção de comportamentos adequados por parte do cuidador (Moreira et al., 2018). Neste sentido, a opção recaiu pela utilização da Teoria das Necessidades Humanas Básicas de Maslow para fundamentar a Tecnologia Educativa, por parecer adequada ao atendimento das necessidades da pessoa idosa no ambiente domiciliar.

Tendo em consideração a escassez de investigações sobre a aplicação de tecnologia educativa para o cuidado de idosos no domicílio, este estudo, sustentado na Teoria das Necessidades Humanas Básicas e nos pressupostos da educação para a saúde, pretende-se analisar a eficácia de uma tecnologia educativa na capacitação de familiares cuidadores de pessoas idosas dependentes em domicílio.

\section{Método}

\section{Participantes}

Participaram neste estudo doze cuidadores informais, que acompanhavam adultos mais velhos com dependência internados numa instituição hospitalar de referência em trauma, no município de Fortaleza-Ceará-Brasil. Os cuidadores enquadraram-se num escalão etário situado entre os dezanove e os cinquenta e três anos, dos quais, oito são mulheres e os demais do sexo masculino; seis estavam casados, três solteiros e três conviviam em união estável; 
sendo que seis cursaram o ensino fundamental (9.ํano de escolaridade), três o ensino médio (12.o ano de escolaridade) e os restantes alfabetizados (2. ano de escolaridade); cinco desempregados e os outros sem ocupação definida; seis sem renda fixa, quatro informaram auferir até um salário mínimo vigente e outros declararam obter de um a três salários mínimos mensais; oito residem em imóvel próprio e quatros em residência arrendada. Quanto à procedência, oito eram de Fortaleza e quatro de outros municípios do Estado do Ceará. Todos os participantes eram familiares de adulto mais velho dependente, situados do primeiro até ao terceiro grau.

O contacto com os cuidadores estabeleceu-se do seguinte modo: na Unidade de Internamento havia 20 cuidadores familiares dos adultos mais velhos hospitalizadas. Com a aquiescência da coordenação da unidade de internamento, os familiares cuidadores foram convidados a participar numa reunião alusiva ao estudo em causa. Todos os cuidadores compareceram à reunião. Nela foram expostos a natureza, objetivo, benefícios da pesquisa e esclarecimentos relativamente às dúvidas expostas sobre as etapas do estudo. Sendo assim, doze concordaram em participar na pesquisa, anuindo através da assinatura do termo de consentimento livre e esclarecido e consentimento pós-informado e, ainda, o compromisso de efetiva participação nos encontros programados. $A$ seguir, aprazou-se as entrevistas em datas e horários, conforme a disponibilidade desses cuidadores e a não interferência nas rotinas relacionadas com os cuidados.

Os critérios de inclusão foram: ser cuidador de adulto mais velho dependente, ter mais de 18 anos e participar nos encontros semanais. Para este estudo seriam excluídos os cuidadores familiares que faltassem a dois dos dez encontros programados. Ressalte-se, contudo, que nenhum participante foi excluído.

Mudanças nas perceções de saúde e envelhecimento sugerem que não há mais pessoa tipicamente velha e que a idade avançada também não implica dependência (WHO, 2015). Porém, para este estudo, considerou-se idoso a pessoa com idade igual ou superior a 60 anos conforme determina o ordenamento jurídico brasileiro, local onde este estudo foi realizado.

\section{Medidas / Instrumentos}

\section{Entrevista}

As entrevistas semiestruturadas foram direcionadas a cada familiar cuidador, com questões que incidiram sobre dados identificativos, tempo dedicado à prestação de cuidados, dificuldades percecionadas no suprimento das necessidades humanas básicas, dependência do adulto mais velho, entre outras. As questões foram previamente elaboradas pelos investigadores que, cumulativamente, realizaram observação participante, e que, com permissão de gravação em áudio, transcreveram as entrevistas integralmente.

\section{Observação Participante}

Realizou-se observação participante como método de recolha de dados. Esta técnica adota como estratégia básica a observação in loco do fenómeno que se pretende compreender (Peruzzo, 2017). A recolha de dados foi concretizada por duas académicas de enfermagem, devidamente integradas no grupo (Mónico et al., 2017) e treinadas para o registo das anotações em diário de campo, conforme a temática de cada sessão. As anotações registadas no diário de campo subsidiaram a recolha de dados a qual foi concluída quando alcançado o princípio da saturação, ou seja, a partir do momento que não emergiram novas ideias relacionadas com o tema em estudo. Nesta aceção, as vivências quotidianas e o senso comum foram elementos de investigação, sendo as linguagens 
que permeiam as experiências pessoais e as interações, os instrumentos conducentes à compreensão do domínio sob investigação, de forma holística e natural.

\section{Procedimento}

Estudo qualitativo, do tipo descritivo e interpretativo, apoiado nos pressupostos metodológicos que suportam a investigação-ação. A recolha de dados foi efetuada no período de novembro a dezembro de 2018, por meio de técnicas de entrevista e de observação participante. A observação participante foi realizada por duas académicas de enfermagem, devidamente treinadas para o registro das anotações consideradas relevantes em diário de campo, conforme a temática de cada encontro. Na entrevista inicial, o foco foi colocado nos dados sócio demográficos dos participantes e no conhecimento prévio sobre os cuidados à ao adulto mais velho dependente. As etapas adotadas para aplicação da Tecnologia Educativa consistiram num encontro prévio para recrutamento dos participantes que incluiu a entrevista inicial, seguida de dez encontros para coleta de dados, sendo dois ou três por semana, com duração média de sessenta minutos, no período da tarde, a fim de não comprometer as atividades realizadas no turno da manhã, e com horário agendado conforme solicitado pelos familiares cuidadores. Ao longo das sessões, os investigadores participantes anotaram no respetivo diário de campo as evidências observadas, consideradas pertinentes e relevantes, que no conjunto das falas extraídas das entrevistas podem concorrer para a fiabilidade e a transferibilidade do estudo para contextos similares.

Esta pesquisa desenvolveu-se de acordo com a Resolução 466/12 da Comissão Nacional de Ética em Pesquisa, que regulamenta a pesquisa com seres humanos sob o parecer favorável do Comitê de Ética do Instituto Dr. José Frota - IJF, sob o n.o 3.001.019/2018. Os cuidadores familiares serão identificados pelas letras FC, seguidas da numeração relativa ao número de participante (FC01 a FC12).

Com consentimento prévio dos participantes, foram efetuadas as entrevistas individuais gravadas que tiveram a duração aproximada de 30 a 40 minutos. Os participantes foram encaminhados para uma sala ampla, silenciosa e climatizada, com cadeiras dispostas em círculo, disponibilizada na Unidade de Internamento, com o intuito de garantir a máxima privacidade e evitar possíveis interrupções durante os encontros. Nesses encontros foram realizadas oficinas educativas através de dinâmicas de grupo, objetivando motivar e fortalecer os vínculos afetivos, favorecendo assim a construção coletiva do conhecimento sobre o cuidado a prestar à pessoa idosa dependente conforme sinopse expressa no Quadro 1.

A pesquisa de campo realizou-se ao longo de três etapas, descritas a seguir.

a) Primeira etapa - Diagnóstico situacional. Nesta reunião, que abrangeu observação participante, houve apresentação dos envolvidos, expostos a natureza e os objetivos do estudo. Em seguida, foi realizada a primeira entrevista para obtenção dos dados sociodemográficos, bem como aceder à experiência no cuidado ao adulto mais velho dependente. Esta etapa finalizou com o agendamento do primeiro encontro para a aplicação da Tecnologia Educativa.

b) Segunda etapa - aplicação da Tecnologia Educativa. O primeiro encontro foi dedicado ao resgate e à socialização da experiência como cuidador do adulto mais velho. Entre o segundo e o nono encontros foram abordadas as práticas concernentes ao atendimento das necessidades humanas básicas, designadamente, necessidades biológicas (nutrição, hidratação, eliminações, sono e repouso, prevenção de risco à vida e ao bem-estar, higiene oral e corporal, e medidas promotoras de saúde), psicossociais (segurança, amor, comunicação, lazer, solidão e interação social) e espirituais (religião e filosofia de vida). 
O décimo e último encontro foi dedicado ao feedback decorrente da participação nos encontros e autoavaliação do familiar cuidador.

c) Terceira etapa - Aplicação de entrevistas de autoavaliação. As entrevistas aconteceram nas datas imediatas à última sessão. Seguiu-se nova entrevista telefónica, gravada mediante o consentimento dos participantes, nos trinta dias subsequentes ao último encontro.

\section{Quadro 1}

Tecnologia Educativa - Capacitação do Familiar Cuidador na Atenção ao Adulto Mais Velho Dependente

\begin{tabular}{|c|c|c|c|c|c|c|}
\hline $\begin{array}{l}\text { Pressuposto da } \\
\text { Educação em } \\
\text { Saúde }\end{array}$ & Conteúdos & Objetivos & $\begin{array}{l}\text { Estratégias } \\
\text { (Dinâmicas) }\end{array}$ & Recursos & Avaliação & Encontros \\
\hline \multirow[t]{8}{*}{$\begin{array}{l}\text { Valorização do } \\
\text { saber construído } \\
\text { pela experiência } \\
\text { vivenciada }\end{array}$} & $\begin{array}{l}\text { Socialização da } \\
\text { experiência do } \\
\text { cuidador na } \\
\text { atenção à saúde } \\
\text { do adulto mais } \\
\text { velho }\end{array}$ & $\begin{array}{l}\text { Estabelecer vínculos entre } \\
\text { os participantes. } \\
\text { Resgatar as experiências do } \\
\text { cuidador com foco na } \\
\text { atenção à saúde do adulto } \\
\text { mais velho }\end{array}$ & $\begin{array}{l}\text { - Pacto de } \\
\text { convivência } \\
\text { - Entrevista } \\
\text { - Balões de } \\
\text { conhecimento }\end{array}$ & $\begin{array}{l}\text { Balões } \\
\text { coloridos, } \\
\text { papel ofício }\end{array}$ & $\begin{array}{l}\text { Questões colocadas ao } \\
\text { grupo para resgate de } \\
\text { experiências }\end{array}$ & 10 \\
\hline & $\begin{array}{l}\text { Necessidades } \\
\text { básicas: nutrição }\end{array}$ & $\begin{array}{l}\text { Discutir sobre o padrão } \\
\text { alimentar e os cuidados } \\
\text { com a administração }\end{array}$ & - O repolho & $\begin{array}{l}\text { Papel ofício, } \\
\text { aparelho de } \\
\text { som }\end{array}$ & $\begin{array}{l}\text { Questões norteadoras } \\
\text { de sensibilização }\end{array}$ & 20 \\
\hline & $\begin{array}{l}\text { Necessidades } \\
\text { básicas: } \\
\text { hidratação }\end{array}$ & $\begin{array}{l}\text { Conscientizar sobre a } \\
\text { necessidade de hidratação } \\
\text { no idoso e a prevenção de } \\
\text { alterações/agravos } \\
\text { associados }\end{array}$ & $\begin{array}{l}\text { - Construindo } \\
\text { conceitos }\end{array}$ & $\begin{array}{l}\text { Cartolinas, } \\
\text { fita crepe, } \\
\text { aparelho de } \\
\text { som }\end{array}$ & $\begin{array}{l}\text { Questões norteadoras } \\
\text { de sensibilização e } \\
\text { resgate do encontro } \\
\text { anterior }\end{array}$ & 3o \\
\hline & $\begin{array}{l}\text { Necessidades } \\
\text { básicas: } \\
\text { eliminação }\end{array}$ & $\begin{array}{l}\text { Discutir sobre as } \\
\text { caraterísticas das } \\
\text { eliminações e alterações no } \\
\text { do adulto mais velho }\end{array}$ & $\begin{array}{l}\text { - Papel amassado } \\
\text { - Brainstorming }\end{array}$ & $\begin{array}{l}\text { Papel ofício, } \\
\text { aparelho de } \\
\text { som }\end{array}$ & $\begin{array}{l}\text { Questões colocadas ao } \\
\text { grupo para discussão e } \\
\text { resgate do encontro } \\
\text { anterior }\end{array}$ & 40 \\
\hline & $\begin{array}{l}\text { Necessidades } \\
\text { básicas: sono e } \\
\text { repouso versus } \\
\text { atividade }\end{array}$ & $\begin{array}{l}\text { Discutir sobre as } \\
\text { caraterísticas e padrão de } \\
\text { sono e repouso articulado } \\
\text { com atividade adequado ao } \\
\text { adulto mais velho }\end{array}$ & $\begin{array}{l}\text { - Construindo } \\
\text { conceitos }\end{array}$ & $\begin{array}{l}\text { Papel ofício, } \\
\text { aparelho de } \\
\text { som }\end{array}$ & $\begin{array}{l}\text { Questões colocadas ao } \\
\text { grupo para discussão e } \\
\text { resgate do encontro } \\
\text { anterior }\end{array}$ & 50 \\
\hline & $\begin{array}{l}\text { Necessidades } \\
\text { básicas: higiene } \\
\text { corporal }\end{array}$ & $\begin{array}{l}\text { Discorrer sobre a } \\
\text { importância da higiene } \\
\text { corporal e oral }\end{array}$ & - O Bingo & $\begin{array}{l}\text { Cadeiras na } \\
\text { sala de } \\
\text { reunião }\end{array}$ & $\begin{array}{l}\text { Questões colocadas ao } \\
\text { grupo para discussão e } \\
\text { resgate do encontro } \\
\text { anterior }\end{array}$ & 60 \\
\hline & $\begin{array}{l}\text { Necessidades } \\
\text { básicas: interação } \\
\text { social e } \\
\text { comunicação } \\
\text { efetiva }\end{array}$ & $\begin{array}{l}\text { Conscientizar o cuidador da } \\
\text { necessidade do adulto mais } \\
\text { velho de interação social e } \\
\text { diálogo }\end{array}$ & $\begin{array}{l}\text { - Construindo } \\
\text { conceitos }\end{array}$ & $\begin{array}{l}\text { Barbante, } \\
\text { óculos } \\
\text { escuros e } \\
\text { luvas }\end{array}$ & $\begin{array}{l}\text { Questões colocadas ao } \\
\text { grupo para discussão e } \\
\text { Resgate do encontro } \\
\text { anterior }\end{array}$ & 70 \\
\hline & $\begin{array}{l}\text { Prevenção do } \\
\text { risco de vida, } \\
\text { bem-estar e } \\
\text { promoção de } \\
\text { saúde e } \\
\text { imunização }\end{array}$ & $\begin{array}{l}\text { Capacitar o cuidador sobre } \\
\text { as condutas preventivas de } \\
\text { risco a vida e promotoras de } \\
\text { saúde e imunização }\end{array}$ & $\begin{array}{l}\text { - Roda de } \\
\text { conversas }\end{array}$ & $\begin{array}{l}\text { Cadeiras na } \\
\text { sala de } \\
\text { reunião }\end{array}$ & $\begin{array}{l}\text { Questões colocadas ao } \\
\text { grupo para discussão e } \\
\text { Resgate do encontro } \\
\text { anterior }\end{array}$ & 8ㅇ e 90 \\
\hline $\begin{array}{l}\text { Empoderamento e } \\
\text { mudança de } \\
\text { comportamento }\end{array}$ & $\begin{array}{l}\text { Avaliação e } \\
\text { autoavaliação de } \\
\text { mudanças na } \\
\text { presença do } \\
\text { cuidado }\end{array}$ & $\begin{array}{l}\text { Proceder com a avaliação e } \\
\text { autoavaliação do cuidador } \\
\text { com vista ao cuidado à } \\
\text { saúde do adulto mais velho }\end{array}$ & - Simulação & $\begin{array}{l}\text { Papel de } \\
\text { ofício }\end{array}$ & $\begin{array}{l}\text { Feedback através de } \\
\text { perguntas ao grupo e } \\
\text { dinâmica de } \\
\text { autoavaliação }\end{array}$ & $10 \circ$ \\
\hline
\end{tabular}


Os dados obtidos foram analisados segundo os pressupostos da análise de conteúdo preconizados por Bardin (2016), designadamente, pré-análise, exploração do material e tratamento dos resultados, inferência e interpretação. A pré-análise foi dedicada à leitura das entrevistas e das anotações constantes nos diários de campo para, assim, diversificar as provas seguindo-se a extração dos recortes das falas dos participantes que foram consideradas relevantes. Posteriormente, procedeu-se à codificação dos dados perfilados no objetivo do estudo dando lugar às categorias temáticas. Portanto, os dados registados pelos observadores participantes e os dados extraídos integralmente da transcrição das entrevistas foram submetidos a análise de conteúdo (Bardin, 2016). Neste processo não foi utilizada nenhuma escala de avaliação para determinar a existência de confiabilidade entre os juízes. Contudo, a análise temática foi realizada por dois dos investigadores que não participaram na recolha de dados e, quando surgiram diferenças na categorização, um terceiro investigador interveio e procedeu ao desempate. A primeira categoria respeita à aquisição de informação mediada pela aplicação da tecnologia educativa; e a segunda às mudanças ocorridas no atendimento às necessidades humanas básicas do adulto mais velho no cuidado domiciliar. A confiabilidade e a concordância entre os investigadores, na codificação e categorização dos dados foi tido em consideração neste estudo. Posteriormente procedeu-se à interpretação dos dados tendo por fundamento a Teoria das Necessidades Humanas Básicas, os pressupostos da educação para a saúde e a literatura de referência.

\section{Resultados}

\section{Aquisição de Conhecimento Mediado pela Aplicação da Tecnologia Educativa}

A participação dos familiares cuidadores nos encontros educativos possibilitou a aprendizagem sobre prevenção de quedas, alimentação, higiene, desenvolvimento da paciência e da tolerância, dedicação, cuidado de qualidade e seleção de atividades de lazer adequadas ao idoso. Os familiares cuidadores identificaram a existência de diversos riscos ambientais a que os idosos estavam expostos. Na tentativa de diminuir estes riscos foram enunciadas estratégias e adaptações, ainda que baseadas no senso comum e nas observações do comportamento do próprio adulto mais velho. A partilha de experiências contribuiu para a compreensão de perigos desconhecidos e estimularam uma mudança imediata no ambiente em que o adulto mais velho está incluído, diminuindo os riscos de quedas e outros acidentes domésticos:

(...) colocar um piso antiderrapante no banheiro, deixar uma luz boa na casa, aprendi foi muito. Quando meu avô adoeceu, minha irmã tomou conta da casa e da cozinha. Minha avó é cega e um dia quase bota fogo na casa. Aprendi a não trocar as coisas de canto na casa da minha avó (...) (FC03, 33 anos).

Após as oficinas, os familiares cuidadores expressaram a necessidade de adequar a alimentação às reais necessidades dos adultos mais velhos, conforme observamos no trecho que segue:

(...) depois da oficina eu tirei algumas comidas que estavam fazendo mal para ele e coloquei vitaminas. Achei o encontro maravilhoso, já contei a todos na enfermaria para ter cuidado quando for comer cuscuz. Aprendi a banhar, dá comida do jeito certo. Acho que dá para ir pra casa agora (...) (FC12, 39 anos). 
A participação do familiar cuidador nas oficinas educativas viabilizou mudanças na forma de cuidar a partir da compreensão do significado de ser cuidador, da aquisição de conhecimentos e da clarificação de dúvidas. Também demonstrou a transição de comportamento do familiar cuidador perante a prestação de cuidados:

(...) Aprendi muito, porque eu tinha muitas dúvidas e agora acabou (...) mais tolerante, sabe? No meu quarto tem um idoso grosso, ajudamos ele e ele sempre é grosseiro, se fosse em outros tempos eu dava na cara dele, mas eu mudei. Acho que agora vou ser outra pessoa, agora eu sei o que é a vida de idoso, gostei muito de aprender. A trocar fralda também, eu tinha dúvida (...) (FC01, 51 anos).

\section{Mudanças Ocorridas no Atendimento às Necessidades Humanas Básicas do Adulto Mais Velho no Cuidado Domiciliar}

A aplicação da tecnologia educativa possibilitou aos familiares cuidadores a aquisição de habilidades e de competências para prestarem cuidados, potenciou o envolvimento da família, aumentou a compreensão das dificuldades, contribuiu para o resgate da autonomia e cooperou para a adequação do ambiente físico adequado ao adulto mais velho. Além disso, o familiar cuidador converteu-se num agente multiplicador das boas práticas de cuidados ao adulto mais velho dependente junto da família:

(...) fiquei mais segura para cuidar dele, eu já cuidava, mas agora sei que estou fazendo o certo e que, quando eu tiver uma dúvida, é só perguntar no posto. O encontro que mais gostei foi essa das pernas amarradas $e$ visão difícil. Não sabia que eles tinham tanta dificuldade pra andar (...) (FC01, 51 anos).

(...) Aprendi muito e se eu não ficar cuidando dele, vou saber quando precisar cuidar dos outros. Foi bom demais. Gostei sobre a água que eles têm que tomar, agora vejo que eu dava bem pouquinha água para ele. Ele não queria tomar, eu não dava. Agora dou de pouquinho, e ele vai tomando gosto de beber mais. Foi tudo bom demais (...) (FCO7, 28 anos).

O uso de diversas estratégias de tecnologia educativa comporta a conscientização do familiar cuidador sobre a importância da autonomia do adulto mais velho e a perceção de que este deve ser participante ativo na manutenção de um estilo de vida saudável, adequado ao seu estado de saúde:

(...) ele é meio reclamão, tem que ficar todo tempo dando atenção, mas já está se recuperando e fazendo as coisas aos poucos. Ele não queria nem comer sozinho, mas aos poucos estamos colocando-o para comer sozinho com as mãos dele. Ele agora está mais sossegado de estar em casa (...) (FC02, 41 anos).

Os familiares cuidadores revelaram a experiência vivenciada após alta hospitalar do adulto mais velho dependente. Após período de permanência hospitalar, esse adulto regressa acompanhado por novas problemáticas que obrigam o cuidador a se adequar a novas rotinas e a gerir dificuldades familiares, económicas e estruturais no domicílio:

(...) afastei os móveis, tirei muito tapete que tinha e alarguei a porta do banheiro e do quarto para passar a cadeira de roda. Não deixo ninguém derramar um copo d'água no chão. Eu já sei como lidar com as zangas dele. Me sinto mais tranquila para mexer com ele, aprendi muito com as dicas dadas (...) (FC09, 39 anos). 
Apesar das inseguranças frequentes relativamente ao retorno do adulto mais velho ao lar, depreende-se que o familiar cuidador que beneficiou da tecnologia educativa está mais confiante nas suas competências para cuidar desse adulto dependente, conforme expressa o seguinte depoimento:

(...) passei tudo pro pessoal da casa dele. E tinha até umas coisas que eles estavam fazendo errado e eu cheguei bem na hora. Eles faziam tudo errado, dava comida dele deitado, deixa o pobre no escuro sem ninguém conversando com ele na cadeira de rodas sozinho, eu até disse para levar ele para calçada para ver o movimento da rua. Aquelas reuniões foram boas pra eu saber dessas coisas (...) (FC05, 34 anos).

Os depoimentos dos familiares cuidadores apoiam a eficácia da tecnologia educativa na capacitação do familiar cuidador para atender às necessidades humanas básicas de adultos mais velhos no cuidado domiciliar.

\section{Discussão}

Este estudo analisa a eficácia de uma tecnologia educativa na capacitação de familiares cuidadores de adultos mais velhos. Os pressupostos subjacentes à construção de uma tecnologia educativa em educação para a saúde foram alavancados na valorização do saber construído a partir da experiência vivenciada, no empoderamento do cuidador relativamente à importância da socialização da experiência do cuidador, no conhecimento das necessidades humanas básicas, na prevenção de riscos e a consequente mudança de comportamento do cuidador familiar de adultos mais velhos no ambiente domiciliar.

O familiar cuidador quando assume a função de cuidar, geralmente, não dispõe de conhecimentos suficientes nem é treinado para desenvolver as novas rotinas que invadem o seu quotidiano. Pautada na necessidade de orientação e de informação do familiar cuidador, a literatura internacional coloca em evidência estratégias exitosas de acompanhamento pós-alta hospitalar como suporte para as necessidades apresentadas pelos cuidadores familiares de idosos dependentes (Aoun et al., 2018; Cardoso et al., 2018; Slatyer et al., 2019). Diante disso, a tecnologia educativa que integra o presente estudo foi construída e aplicada com a finalidade de favorecer a aprendizagem de saberes antes desconhecidos, ampliar a possibilidade de compreensão sobre o complexo processo de envelhecimento, ajudar à construção da tolerância e, em suma, reunir subsídios para ampliar a capacitação do familiar cuidador do adulto mais velho com dependência funcional após a alta hospitalar. As estratégias educacionais atinentes ao cuidado a esse adulto no domicílio, promotoras da aquisição de conhecimento, segundo o depoimento dos participantes, foram reconhecidas como fundamentais. Os resultados do presente estudo apoiam a ideia de que a tecnologia educativa aplicada contribuiu para gerar nos participantes a consciência da situação real do idoso dependente e da efetivação do cuidar (José et al., 2016), superando uma perspetiva meramente técnica e abstrata (Cardoso et al., 2018).

As dificuldades da família, na prestação do cuidado ao adulto mais velho, estão associadas ao enfrentamento do adoecimento face a incapacidades e/ou à dependência total ou parcial para a realização das necessidades básicas. Este fato agrava-se devido ao défice de orientações e de saberes, e consequentemente, gera ansiedade e crenças de ineficácia nem relação à prestação do cuidado ao adulto mais velho. Assim sendo, as intervenções educativas centradas na capacitação do cuidador, envolvendo escuta ativa, integração do cuidador em grupos de apoio, tornam-se suscetíveis de impactarem a práxis do familiar cuidador na realização do cuidado domiciliar ao idoso. A tecnologia educativa também emerge como recurso essencial para o esclarecimento de dúvidas, partilha de 
experiências e de angústias, podendo concorrer para a redução dos níveis de stresse e auxiliar na adaptação à nova realidade (Ministério da Saúde, 2012).

Os familiares cuidadores expressaram preocupação relativamente aos riscos ambientais que podem afetar os adultos mais velhos. Na tentativa de diminuição dos riscos, referiram a utilização de condutas preventivas de riscos de vida, tendentes à diminuição de quedas e/ou outros acidentes domésticos e promotoras da saúde do idoso. Tal preocupação é consentânea com a literatura quando evidencia que a ocorrência de quedas se dá, principalmente, dentro do ambiente doméstico (Luiz et al., 2015) e reforça a necessidade de adaptações ergonômicas no ambiente domiciliar da pessoa idosa, para que possa estar integrada num local seguro, em que o potencial risco de quedas seja diminuto (Marinho et al., 2020). Neste contexto, os participantes destacaram a necessidade de redimensionamento dos espaços destinados à instalação do adulto mais velho. dependente ou não no domicílio, supressão de barreiras arquitetónicas obstrutivas da locomoção do idoso, além da aquisição de meios compensatórios, designadamente, corrimões e alças de apoio, leitos com elevação ou rebaixamento, cadeiras de banho, cadeiras de rodas, entre outros, visando promover a adaptação do adulto mais velho, facilitar a prestação dos cuidados e evitar o agravamento da saúde do idoso, por exemplo, com o risco de quedas.

No respeitante a aspetos citados nos achados, concernentes ao suprimento das necessidades básicas do adulto mais velho, os participantes enunciaram a nutrição e a higiene como mais preocupantes. No processo de envelhecimento, acontecem alterações que podem influenciar a mastigação e a deglutição, obrigam novas adaptações centradas na elaboração cuidada e personalizada do plano alimentar visando assegurar um bom estado nutricional do adulto mais velho, nesta etapa da vida. A capacitação dos familiares cuidadores sobre domínios relacionados com as peculiaridades inerentes às mudanças fisiológicas do envelhecimento auxilia na manutenção de um bom estado nutricional da pessoa cuidada. Esta evidência faz parte do cotidiano de cuidadores formais (Cunha, et al., 2020) e, por isso, durante a aplicação da tecnologia educativa, foi desenvolvido um vídeo educativo alusivo à alimentação específica do idoso. Neste âmbito, as tecnologias educativas, em formato impresso ou on-line, desenvolvidas com os cuidadores, enquanto instrumentos dinamizadores, contribuem para a capacitação do cuidador e, pelo efeito coletivo que imprimem, também cooperam na orientação e informação da população e, ainda, dos profissionais prestadores de cuidados a idosos (Cardoso et al., 2018). Os resultados evidenciaram o empenho dos familiares cuidadores na aquisição de competências conducentes à prestação de cuidados com qualidade. Para tal concorreu o incentivo dado pela estratégia de educação em saúde, que almeja diminuição das dificuldades e incentivo da continuidade de prestação do cuidado (Santos et al., 2018).

Ainda sob a ótica do cuidado, é importante refletir acerca da compreensão por parte do familiar cuidador relativamente às alterações biológicas, psicológicas, físicas, mentais e como elas interferem na conduta da pessoa idosa, bem como, o conhecimento das consequências danosas trazidas quando não são atendidas as necessidades humanas básicas do adulto mais velho. $O$ processo de envelhecimento é sentido por cada indivíduo de forma heterogênea e subjetiva. Diversos fatores biológicos, sociais, socioculturais e estruturais incidem diretamente no curso de vida individual, possibilitando um envelhecimento saudável ou, em contrapartida, malsão. A vulnerabilidade das pessoas idosas no processo de envelhecimento é evidenciada tanto pela fragilidade psicológica quanto física, impactando diretamente a autonomia do idoso e a perceção/vivência da mesma no seu cotidiano (Miranda et al., 2015). Com base nos depoimentos dos familiares cuidadores é notória a perceção dos familiares cuidadores relativamente à necessidade de desenvolvimento da autonomia do adulto mais velho, da importância do seu papel ativo no processo decisório e do respeito pelas suas opções. Neste sentido, Paschoal 
(2008) advoga que o estímulo ao autocuidado é fundamental, e que os cuidados só devem ser realizados pelo cuidador quando a pessoa idosa não conseguir realizá-los.

Fragilidades face aos efeitos deletérios afetivos, sociais, psicológicos e fisiológicos do idoso, durante o período de hospitalização e afastamento do lar são percecionados pelo familiar cuidador. Após a alta hospitalar, no retorno ao domicílio, os cuidados passam a ser desenvolvidos pela própria família (Oliveira et al., 2011). Esta depara-se com uma nova realidade, confrontada com uma diversidade de tarefas complexas para as quais não está preparada, que por vezes as realizava intuitivamente, e que alteram a dinâmica pessoal, familiar, profissional e social (Castro et al., 2016; Fernandes \& Angelo, 2016). Além disso, a prestação de cuidados impõe sobrecarga ao cuidador, pois, este dedica-se por um longo período ao adulto mais velho na atenção às necessidades básicas que envolvem múltiplos domínios, designadamente, cuidado físico, mobilização, alimentação, apoio emocional, entre outras (Marigliano et al., 2015). Portanto, cuidar de um idoso dependente no domicílio acarreta grande ônus ao cuidador e à sua família. Este ônus está vinculado ao nível físico, psíquico, social e financeiro. Em função disso, a Equipa de Saúde tem um papel crucial na atenção a quem cuida, visando a promoção da sua saúde e bem-estar, bem como no encaminhamento para busca de suporte social e recursos financeiros. Isto porque, um cuidador familiar bem informado é capaz de enfrentar, com maior segurança, os desafios cotidianos impostos pelo ato de cuidar (Miranda et al., 2015).

No presente estudo, a aplicação da tecnologia destinada à capacitação do familiar cuidador na atenção ao adulto mais velho, ancorada no atendimento às necessidades humanas básicas da pessoa idosa no cuidado domiciliar, imprimiu mudança atitudinal, reforçou estratégias tendentes à preservação da autonomia da pessoa idosa e estimulou a participação ativa no processo de cuidar, corroborando com o estudo de Pacheco et al. (2020). Refletindo sobre os achados deste estudo, pesquisas evidenciam que ações educativas sensibilizam e promovem mudanças comportamentais e atitudinais nos familiares cuidadores (Cardoso et al., 2018; Landeiro et al., 2016; Pacheco et al., 2020; Santos et al., 2018). Assim sendo, a implementação de práticas educativas focadas nos familiares cuidadores tem-se convertido num contributo significativo na prevenção de riscos, de agravos e incapacidades e, sobretudo, na melhoria da qualidade de vida e do bem-estar do idoso. Portanto, a educação em saúde não se configura obsoleta tecnologicamente, uma vez que o resultado da aplicação de tecnologias educativas tem se revelado grandemente impactante e modificadora de atitudes quando aplicada de forma científica e sistematizada. Tendo em conta a importância da informação e educação em saúde para os cuidadores familiares e o impacto da aplicação de tais saberes no bem-estar da pessoa cuidada, diversos países criaram legislação visando mitigar as vulnerabilidades inerentes ao processo de cuidar. Por exemplo, Portugal e suas Regiões Autónomas dos Açores e da Madeira aprovaram normativos concernentes à criação do estatuto do cuidador informal, que regulam os direitos e os deveres do cuidador e da pessoa cuidada, e estabelecem as respetivas medidas de apoio (Decreto Legislativo Regional n.o 22/2019/A, de 29 de novembro, 2019; Decreto Legislativo Regional n.o 5/2019, de 17 de julho, 2019; Lei n.o 100/19). Também, no Reino Unido (Care Act, 2014), foi consagrada legislação de aconselhamento, registo e capacitação do cuidador, exigindo que os hospitais identifiquem cuidadores familiares, os quais são informados sobre os planos para alta do paciente e formados para a prestação de cuidados.

Ressalta-se a falta de avaliação prévia dos níveis de literacia em saúde dos cuidadores, maioritariamente com baixa escolarização, o que condiciona geralmente a disponibilidade para a aprendizagem. Além disso, neste estudo, a falta de adesão de diversos cuidadores familiares configura uma das limitações. Um número maior de participantes poderia aportar mais informações, expor mais problemáticas e desencadear novas soluções. Além 
disso, o estudo foi realizado apenas numa instituição hospitalar de referência em traumas, no Ceará, Brasil, mas crê-se que a tecnologia pode ser transferível para contextos socioculturais semelhantes.

Mediante as múltiplas vantagens e do impacto significativo da tecnologia educativa apresentada na capacitação e na vida do familiar cuidador e, sobretudo, na satisfação das necessidades humanas básicas do adulto mais velho dependente, e na perspetiva humanista em que o respeito pela dignidade humana se impõe, é de considerar a importância da inserção de competências geriátricas e gerontológicas nos currículos da escolarização obrigatória e, sobretudo, em todos os currículos dos cursos associados à saúde.

\section{Conclusão}

Os resultados deste estudo permitem concluir que a tecnologia educativa é uma ferramenta que pode ser utilizada como complemento útil para reforçar as competências do cuidador informal de adultos mais velhos dependente. Promove o conhecimento sobre o processo de envelhecimento ao nível da dependência funcional e capacita os cuidadores informais para a prestação de cuidados tendentes à satisfação das necessidades humanas básicas. Concomitantemente, a aplicação dos saberes mediados pela tecnologia permite prevenir doenças e agravos, adotar procedimentos adequados à situação do adulto mais velho, ampliar os níveis de tolerância, potenciar a busca de soluções para os problemas e, em suma, ajudar a suprir as necessidades humanas básicas do adulto mais velho. Neste domínio, a tecnologia educativa promove a consciencialização de que a prestação de cuidados assente na satisfação das necessidades humanas básicas, que é uma resposta adequada e possível nas situações que envolvem a prestação de cuidados aos adultos mais velhos dependentes em ambiente domiciliar.

Conflito de interesses | Conflict of interest: Nenhum | None.

Fontes de financiamento | Funding sources: Nenhuma | None.

Contributos: LPRO: Contributo na conceção do tema do estudo; Definição da metodologia, coleta dos dados, análise e interpretação dos indicadores e elaboração do manuscrito; Revisão crítica e aprovação da versão final do artigo. ZMSAS: Contributo na conceção do tema do estudo; Definição da metodologia, coleta dos dados, análise e interpretação dos indicadores e elaboração do manuscrito; Revisão crítica e aprovação da versão final do artigo. MRTFC: Contributo na redação, na revisão crítica, na aprovação da edição final do manuscrito. JGOB: Contributo na conceção do tema do estudo; Definição da metodologia, coleta dos dados, análise e interpretação dos indicadores e elaboração do manuscrito; Revisão crítica e aprovação da versão final do artigo. EPRO: Contributo na conceção do tema do estudo; Definição da metodologia, coleta dos dados, análise e interpretação dos indicadores e elaboração do manuscrito; Revisão crítica e aprovação da versão final do artigo. MPSF: Contributo na redação, na revisão crítica, na aprovação da edição final do manuscrito.

\section{Referências}

Alves, N. M. (2018). Cuidando, lado a lado - Otimização de estratégias de atuação na capacitação do cuidador informal [Tese de mestrado, Universidade de Évora]. Repositório da Universidade de Évora. https://bit.ly/3fh8FMj

Aoun, S. M., Stegmann, R., Slatyer, S., Hill, K. D., Parson, R., Moorin, R., Bronson, M., Walsh, D., \& Toye, C. (2018). Hospital postdischarge intervention trialled with family caregivers of older people in Western Australia: Potential translation into practice. BMJ Open, 8(11), Artigo e022747. https://doi.org/gdvr

Bardin, L. (2016). Análise de conteúdo (L. Antero Reto, Trad.). Edições 70.

Cardoso, R. S. S, Sá, S. P. C, Domingos, A. M, Sabóia, V. M, Maia, T. N., Padilha, J. M. F., \& Nogueira, G. A. (2018). Educational technology: a facilitating instrument for the elderly care. Revista Brasileira de Enfermagem, 71(Supl. 2), 786-92. https://doi.org/gdvt 
Care Act 2014. (2014). Care Act 2014 c. 23. Legislation.gov.uk. https://bit.ly/3hMuOnz

Castro, L., Souza, D., Pereira, A., Santos, E., Lomeo, R., Teixeira, H. (2016). Competências dos cuidadores informais familiares no autocuidado: Autoestima e suporte Social [Apresentação de artigo]. Quinto Congresso IberoAmericano em Investigação Qualitativa (Vol. 2, pp. 1346-1355), Porto, Portugal. https://bit.ly/2QR9ZMG

Ceccon, R. F., Vieira, L. J. E. S. Brasil, C. C. P., Soares, K. G., Portes, V. M., Garcia Júnior, C. A. S., Schneider, I. J. C., \& Carioca, A. A. F. (2021). Envelhecimento e dependência no Brasil: características sociodemográficas e assistenciais de idosos e cuidadores. Ciência \& Saúde Coletiva, 26(1) 17-26. https://doi.org/gfmh

Cruz, D. C. M., Loureiro, H. A. M., Silva, M. A. N. C. G. M. M., Fernandes, \& M. Mouronho (2010). As vivências do cuidador informal do idoso dependente. Revista de Enfermagem Referência, III(2), 127-136. https://doi.org/gfmf

Cunha, D. G. P., Almeida, L. N. A., Wanderley, R. M. M., Bittencourt, G. K. G. D., Alves, G. a. S., Amaral, A. K. F., \& Bezerra, R. G. S. (2020). Alimentação e Comunicação: Vídeo para Orientação de Cuidadores de Idosos. Revista Brasileira de Ciências da Saúde, 24(1), 147-158. https://doi.org/gdvv

Decreto Legislativo Regional n.o 22/2019/A, de 29 de novembro. (2019). Regime jurídico de Apoio ao Cuidador Informal na Região Autónoma dos Açores. Diário da República, 1. a série, 212, 22-29. https://bit.ly/34b91xK

Decreto Legislativo Regional n. 5/2019, de 17 de julho. (2019). Cria o estatuto do cuidador informal da região Autónoma da Madeira. Diário da República, 1. série, 135, 17-22. https://bit.ly/2SIYDke

Fechine, B. R. A., \& Trompieri, N. (2015). O processo de envelhecimento: as proncipais alterações que acontecem com o idoso com o passar dos anos. InterSciencePlace, 1(20), 106-194. https://bit.ly/3fLiyki

Fernandes, C. S., \& Angelo, M. (2016). Cuidadores familiares: o que eles necessitam? Uma revisão integrativa. Revista da Escola de Enfermagem da USP, 50(4), 675-682. https://doi.org/gdvw

Galado, M. (2018). Serpa promove a qualidade de vida dos cuidadores informais! [Tese de mestrado, Universidade de Évora]. Repositório Universidade de Évora. https://bit.ly/3bOx5dY

Horta, W. A. (1978). Processo de enfermagem. EPU.

José, D. G., Schwalm, M. T., Ceretta, L. B., Dagostim, \& V. S., Soratto, M. T. (2016). Adaptação da família do idoso no cuidado domiciliar. Revista Saúde, 16(43), 1-11. https://doi.org/gdv2

Landeiro, M. J., Peres, H. H. C., \& Martins, T. V. (2016). Evaluation of the educational technology "Caring for dependent people" by family caregivers in changes and transfers of patients and tube feeding. Revista Latino-Americana de Enfermagem, 24, Artigo e2774. https://doi.org/gdv3

Lei n.o 100/19, de 6 de setembro. (2019). Aprova o Estatuto do Cuidador Informal, altera o Código dos Regimes Contributivos do Sistema Previdencial de Segurança Social e a Lei n.o 13/2003, de 21 de maio. Diário da República, 1. - série, 171, 3-16. https://bit.ly/3fcUKqB

Luiz, I. C., \& Brum, A. K. R. (2015). Prevalência e fatores de risco de queda em idosos no domicílio: revisão integrativa da literatura. Revista de Enfermagem UFPE on line, 9(10), 1556-1564. https://bit.ly/3fHjXIS

Marigliano, R. X., Silva J. F., Miranda, M. L. J., Rodrigues, G. M., \& Gil, C. A. (2015). Estratégias de autocuidado usadas por cuidadores de idosos: análise de produção científica. Mudanças Psicologia da Saúde, 23(2), 37-45. https://bit.ly/3ff9OE8

Marinho, C. L., Nascimento, V., Bonadiman, B. S. R, \& Torres, R. S. F. (2020). Causas e consequências de quedas de idosos em domicílio. Brazilian Journal Health Review, 3(3), 6880-6896. https://doi.org/gdv8

Melo, L. P. (2016). É como uma família: significados atribuídos a grupos de educação em saúde sobre diabetes por profissionais da saúde. Ciência \& Saúde Coletiva, 21(8), 2497-2506. https://doi.org/gdwb

Ministério da Saúde. (2012). Caderno de atenção domiciliar (Vol. 1). Ministério da Saúde. https://bit.ly/3hKV69l

Ministério da Saúde. (2008). Guia prático do cuidadorå. Ministério da Saúde. https://bit.ly/3fiU8jr

Miranda, A. C. C., Sérgio, S. R., Fonseca, G. N. S., Coelho, S. M. C., Rodrigues, J. S., Cardoso, C. L. C., \& Cassiano, J. G. (2015). Avaliação da presença de cuidador familiar de idosos com déficits cognitivos e funcional residentes em Belo Horizonte-MG. Revista Brasileira de Geriatria e Gerontologia, 18(1), 141-150. https://doi.org/gdwc

Mónico, L., Alferes, V., Parreira, P., \& Castro, P. A. (2017). A observação participante enquanto metodologia de investigação qualitativa [Apresentação de Artigo]. Sexto Congresso Ibero-Americano em Investigação Qualitativa (Vol. 3, pp. 724-733), Salamanca, Espanha. https://bit.ly/3oXmdzZ

Moraes, S. L. M, Tabelo, M. B. D., Afio, C. J., Lavinas, S. M. C., \& Santos, A. M. D. (2016). Uso de tecnologia leve-dura nas práticas de enfermagem: análise de conceito. Aquichan, 16(2), 230-239. https://doi.org/gdwd 
Moreira, A. C. A., Silva, M. J., Darder, J. J. T., Coutinho, J. F. V., Vasconcelos, M. I. O., \& Marques, M.B. (2018). Effectiveness of an educational intervention on knowledge-attitude-practice of older adults' caregivers. Revista Brasileira de Enfermagem, 71(3), 1055-1062. https://doi.org/gdwf

Oliveira, B. C., Garanhani, M. L., \& Garanhani, M. R. (2011). Caregivers of people with stroke - needs, feelings and guidelines provided. Acta Paulista de Enfermagem, 24(1), 43-49. https://doi.org/gdwg

Organização Pan-Americana da Saúde/Organização Mundial de Saúde. (2018). Folha Informativa-Envelhecimento e Saúde. Organização Pan-Americana da Saúde/Organização Mundial de Saúde. https://bit.ly/3oQfZ4H

Pacheco, E. S., Rocha, K. A., Mota, M. S., Silva, V. R, Gomes, A. T., Viana, V. M. O., Rocha, A. F., \& Cardoso, A.R. (2020). Perceptions of elderly caregivers about the act of caring. Research, Society and Development, 9(7), Artigo e283974161. https://doi.org/gdwj

Paschoal, S. M. P. (2008). Diminuição da capacidade funcional, fragilização e dependência. Em Born, T. (Org.), Cuidar melhor e evitar a violência - Manual do cuidador da pessoa idosa (pp. 113-120). Secretaria Especial dos Direitos Humanos, Subsecretaria de Promoção e Defesa dos Direitos Humanos.

Pereira, S., \& Duque, E. (2017). Cuidar de idosos dependentes - A sobrecarga dos cuidadores familiares. Revista Kairós - Gerontologia, 20(1), 187-202. https://doi.org/gfmg

Pereira, K. C., Guimarães, F. S, Alcauza, M. T. R., Campos, D. A., \& Pires, R. O. M. (2014). Percepção, conhecimento e habilidades de cuidadores em saúde bucal de idosos acamados. Saúde \& Transformação Social, 5(3), 34-41. https://bit.ly/3yzNbln

Peruzzo, C. M. K. (2017). Pressupostos epistemológicos da pesquisa participativa: da observação-participante à pesquisa-ação. Estudios sobre las Culturas Contemporáneas, XXIII(3), 161-190. https://bit.ly/3oZXMBJ

Santos, A. C, Silva, J. O. M., Makuch, M. V., Matia, G., \& Rozin, L. (2017). Sobrecarga do cuidador familiar do idoso dependente. Espaço para a Saúde - Revista de Saúde Pública do Paraná, 18(2), 55-62. https://bit.ly/34aaBA2

Santos, P. D. S., Santos, Z. M. S., Diógenes, L. M. M. B., Caldas, J. M. P., Rodrigues, K. A. F., \& Carneiro, R. F. (2018). Capacitação do familiar cuidador com a aplicação da Tecnologia Educativa em Saúde. Revista Brasileira de Enfermagem, 71(3), 1202-1201. https://doi.org/gdwq

Slatyer, S., Aoun, S. M., Hill, K. D., Walsh, D., Whitty, D., \& Toye, C. (2019). Caregivers' experiences of a home support program after the hospital discharge of an older family member: A qualitative analysis. BMC Health Services Research, 19(1), 220. https://doi.org/gdwr

Sousa, S. G., Silva, R. M., Reinaldo, A. M. S., Soares, S. M., Gutierrez, D. M., \& Figueiredo, M. L. F. (2021). “A gente não é de ferro": Vivências de cuidadores familiares sobre o cuidado com idosos dependentes no Brasil. Ciência \& Saúde Coletiva, 26(1), 27-36. https://doi.org/gfmj

World Health Organization. (2015). World report on ageing and health. WHO Library. https://bit.ly/3hPEUUB 